\title{
Genèses du Tour through Italy de Stendhal (1811-1813)
}

\section{Catherine Mariette-Clot}

\section{(2) OpenEdition}

\section{Journals}

Édition électronique

URL : http://journals.openedition.org/genesis/549

DOI : 10.4000/genesis.549

ISSN : 2268-1590

\section{Éditeur :}

Presses universitaires de Paris Sorbonne (PUPS), Société internationale de génétique artistique littéraire et scientifique (SIGALES)

\section{Édition imprimée}

Date de publication : 1 janvier 2011

Pagination : 195-204

ISBN : 978-2-84050-749-9

ISSN : 1167-5101

\section{Référence électronique}

Catherine Mariette-Clot, « Genèses du Tour through Italy de Stendhal (1811-1813) », Genesis [En ligne], 32 | 2011, mis en ligne le 17 juin 2013, consulté le 19 avril 2019. URL : http://journals.openedition.org/ genesis/549; DOI : 10.4000/genesis.549 


\title{
Genèses du Tour through Italy de Stendhal (1811-1813)
}

\author{
Catherine Mariette-Clot
}

\author{
À Christine et Laurent Arbelet
}

$\mathrm{L}$ 'heureux incident qui a présidé à ce travail fut la redécouverte, au printemps 2008, d'une des strates du dossier génétique du Tour through Italy de Stendhal, perdue depuis 1911. Il s'agit d'une des mises au net réalisées en 1813 par un copiste (Fougeol) à partir d'un cahier autographe de 1811. Cette copie, intitulée de la main de Stendhal « Fin du tour d'Italie, en 1811 », est cousue en cahier, certainement par les soins de celui-ci (voir fig. 1).

Ce cahier connaît une étrange destinée : il fut d'abord légué par la famille de Romain Colomb, le cousin de Stendhal, à Auguste Cordier qui, dans les années 1889-1890, transmet l'ensemble de sa collection de manuscrits autographes à Casimir Stryienski, l'un des premiers «stendhaliens » du nom. Mais Auguste Cordier ne se défait pas de ces feuillets sans en avoir au préalable réalisé de sa propre main une copie en « fac-similé »: il imite l'écriture de Stendhal, transpose soigneusement ses croquis sur papier-calque et les joint à sa transcription (Bibliothèque municipale de Grenoble, R 90729 (2) Rés.). Ensuite, la copie de Fougeol et celle de Cordier disparaissent : en 1972, la bibliothèque de Clermont-Ferrand signale qu'elle possède la copie de Cordier et la bibliothèque municipale de Grenoble en fait l'acquisition. En 1981, dans sa notice à l'édition de la Pléiade, Victor Del Litto saluait cette trouvaille - ou plutôt ces retrouvailles - mais indiquait qu'on ne savait pas où avait disparu le cahier original que Stryienski avait eu entre les mains et que Paul Arbelet avait consulté, affirmait-il ${ }^{1}$. Il devait se trouver dans les mains de particuliers... Effectivement, Paul Arbelet avait consulté ce document pour en faire une première édition avec Stryienski dans les Soirées du Stendhal-Club, Deuxième série, 1908, sous le titre «Fin du Tour d'Italie en 1811 (Cahier complémentaire du "Journal")2 », puis pour son édition du Journal d'Italie, en 19113. Le petit-fils de Paul Arbelet, Laurent Arbelet, venait de découvrir, au printemps 2008, ce précieux cahier dans une malle, au milieu de notes de cours et autres papiers personnels de son grand-père, avec une mention de la main de celuici : «manuscrit de Stendhal ». C'est ainsi que l'équipe « Manuscrits de Stendhal ${ }^{4}$ » fut alertée et que, grâce à la générosité de Laurent Arbelet, je découvris avec émotion un cahier dans l'état où Stendhal l'avait laissé de son vivant, à l'état de nature pourrait-on dire, et jamais encore archivé par les bibliothécaires.

À partir de cet élément retrouvé, j’ai voulu reconstituer tous les maillons de la chaîne écrite, de 1811, point de départ de l'écriture sous la forme particulière d'un « journal de voyage », jusqu'aux ultimes traces visibles dans l'œuvre publiée, au-delà même de ce qui constituait sa borne visible, à savoir les copies de 1813 . Je me poserai ainsi la question de la valeur de l'écriture personnelle dans la mesure où elle est remaniée, dictée, corrigée et qu'elle subit une transformation générique que seule l'étude transversale du dossier génétique peut aider à comprendre. Enfin, je m'intéresserai au statut du brouillon : pourquoi Stendhal, après s'être donné tant de mal à retravailler certaines pages de son journal, les abandonne-t-il ? Le brouillon dans ce cas est-il une impasse, la marque d'une irrésolution ou au contraire une étape vers un futur texte qui l'englobe et le dépasse ?

\footnotetext{
1. Stendhal, Euvres intimes, t. I, éd. Victor Del Litto, Paris, Gallimard, coll. «Bibliothèque de la Pléiade », 1981, p. 1417-1421.

2. Soirées du Stendhal-Club, Deuxième série, Documents inédits, Paris, Mercure de France, 1908, p. 99-144.

3. Journal d'Italie, éd. Paul Arbelet, Paris, Calmann-Lévy, 1911.

4. Équipe «Manuscrits de Stendhal », Centre d'études stendhaliennes, Traverses 19-21, EA 3748, Université Stendhal, Grenoble.
} 


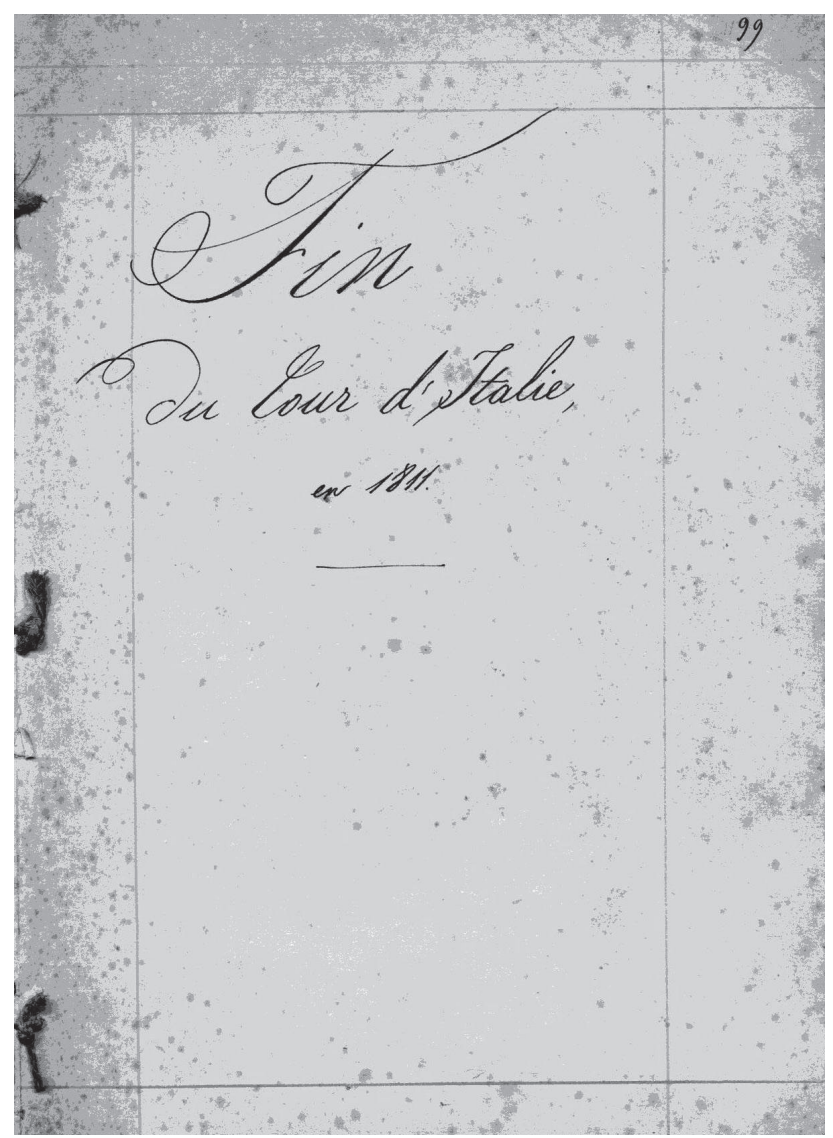

Fig. 1 : Manuscrit $\operatorname{Arbelet}^{5}, \mathrm{f}^{\circ} 99 \mathrm{r}^{\circ}$ (coll. particulière)

\section{Chronologie du Voyage}

Quand il entreprend la rédaction du Voyage en Italie de 18116, Stendhal, auditeur au Conseil d'État, obtient de Pierre Daru, pour lequel il travaille alors, un congé pour l'Italie. Quelques jours avant son départ, il commence à écrire son journal sur un cahier autonome ayant pour titre «A Tour Throught (sic) Italy. $1811 »$ (voir fig. 2). Il y récapitule les motivations et les conditions du voyage :

25 th August 1811

[...] L'idée me vint de demander un congé et d'aller voir Naples et Rome. Je fis ma demande à [un blanc] qui l'accueillit avec une bonté parfaite.
Le « journal de voyage » commence donc à Paris, le 25 août 1811, dans la continuité du journal proprement dit ${ }^{7}$, par le récit des préparatifs et des adieux qu'il fait à Mme Daru et à Angeline Beyreter. Avant d'atteindre l'Italie, le diariste traverse la Bourgogne, la FrancheComté, la Suisse et, quand il arrive enfin à Milan, le 8 septembre, il est bien en Italie mais il ne voyage plus : il « livre bataille » à la belle Angela Pietragrua qu'il désire depuis son premier voyage en Italie de 1800 ; le 20 septembre, la « victoire remportée » fait de Milan le point d'aimantation du récit (il aura du mal à quitter Angela et n'aura de cesse de rentrer à Milan pour la retrouver). Enfin, le 21 septembre, le lendemain de cette victoire, il entame son tour d'Italie proprement dit jusqu'au 24 octobre où il retrouve Angela à Varese ; il reprend alors une nouvelle fois la narration de ses aventures amoureuses. Le 7 novembre, le récit est interrompu, de manière un peu abrupte, sur des considérations esthétiques à peine rédigées, sous forme de notes, alors qu'il ne rentre à Paris que le 27 novembre. On voit donc que ce Tour est autant récit de voyage que récit des amours du diariste, itinéraire personnel autant que culturel.

Le titre, calligraphié de manière cérémonieuse sur la première page du Tour (voir fig. 1), annonce donc un contenu légèrement décalé par rapport à celui qu'on lit effectivement, qui se cherche, s'égare en des pages discontinues où alternent des descriptions de paysages et de tableaux vus dans des musées, des notes de lecture, des réflexions personnelles et des prouesses sexuelles à

5. Nous appelons « Manuscrit Arbelet » ce cahier retrouvé par Laurent Arbelet dans les papiers de son grand-père et mis généreusement à notre disposition. Nous le remercions chaleureusement ici de l'aimable autorisation de reproduire les images qu'il nous a confiées.

6. Il s'agit d'un des titres que Stendhal donna à son « Voyage » (R 5896 (14) Rés., f ${ }^{\circ} 189$ r $^{\circ}$ ) (voir supra).

7. Le voyage est déjà au cœur de l'entrée précédente du 18 août, sous forme de dialogue rapporté avec Mme Daru, qu'il doit quitter à cette occasion : «Il faut absolument que je fasse ce voyage. Je vous aime passionnément ; vous, vous ne voulez pas m'aimer. D'ailleurs your husband me voit de mauvais œil. Je m'en suis aperçu dimanche. Je ne pourrai plus vous voir aussi souvent [...]. Peut-être mon absence arrangera-t-elle tout, et à mon retour me reverra-t-il volontiers »(Euvres intimes, t. I, op. cit., p. 714). 
demi voilées. Le titre affiche une intention littéraire que l'écriture au jour le jour déçoit : le geste emphatique qui le trace montre la solennité d'une amorce scripturaire que dément le contenu du journal qu'on lit, fait de notes souvent elliptiques et fragmentaires. Il renvoie sans doute à une pratique qui s'intensifie depuis la fin du XVIII' siècle et que Chateaubriand a consacrée par son Itinéraire de Paris à Jérusalem, paru en 1811, l'année même où Stendhal amorce son Tour through Italy. Le Journal ne peut échapper à ce «fait culturel 8 » - un voyage donne lieu à sa narration - et l'effet d'annonce que constitue la calligraphie du titre se situe certainement dans ce genre de projet alors dans l'air du temps.

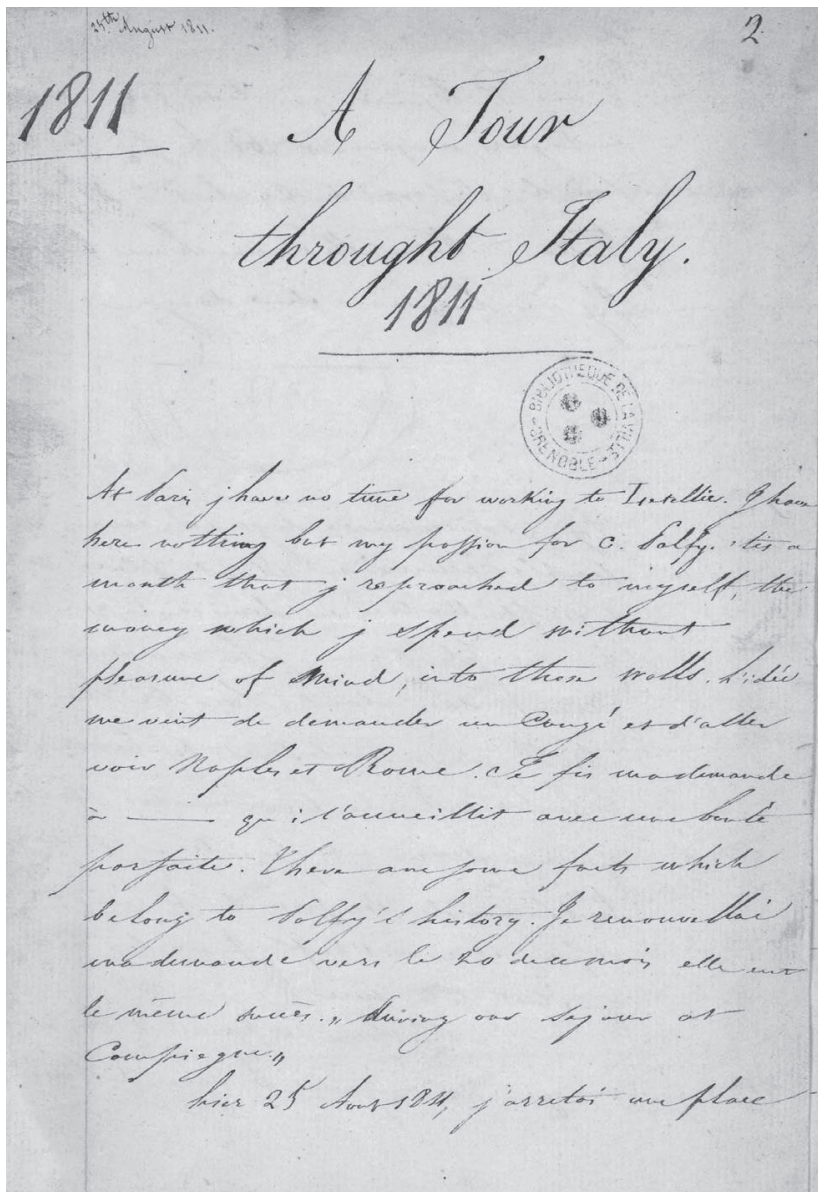

Fig. 2 : Stendhal, «A Tour Throught Italy. $1811 », \mathrm{f}^{\circ} 2 \mathrm{r}^{\circ}$ (R 5896 (23) Rés., Bibliothèque municipale de Grenoble ${ }^{9}$ )

\section{Description matérielle du dossier génétique}

Il s'agissait avant toute interprétation de remettre de l'ordre dans les différentes strates du dossier manuscrit, d'en rétablir la chronologie et, comme nous le suggère Almuth Grésillon, « de restituer le principal fil conducteur de l'écriture, celui qui permet d'ordonner les matériaux selon les lois d'une filiation linéaire, celles qui président aux arbres de la généalogie, bref, les lois du "stemma" » 10

Les éléments du Tour through Italy se déploient ainsi autour de deux grands ensembles temporels : la rédaction du premier jet de 1811 qui épouse le déroulement du voyage réel et la campagne de relecture en 1813 .

Nous avons ainsi deux massifs d'écriture distincts :

\section{Les autographes de 1811}

- un cahier autographe intitulé A Tour Throught (sic) Italy. 1811 (Bibliothèque municipale de Grenoble, R 5896 (23) Rés. ${ }^{11)}$ qui va jusqu'à la date du « 19 8bre 1811 » [19 octobre 1811] (R 5896 (23) Rés., fos $113 \mathrm{r}^{\circ}-114 \mathrm{v}^{\circ}$ ). Relu et corrigé en 1813 : annotations autographes et notes de régie ;

- un second cahier autographe (R 5896 (14) Rés., fos $189 \mathrm{r}^{\circ}-212 \mathrm{r}^{\circ}$ ) qui relate le second séjour à Milan à partir du moment où Henri Beyle retrouve Angela (24 octobre 1811) et qui comporte quatre pages de titre : Collection complète du Voyage en Italie de 1811 ( $\left.\mathrm{f}^{\circ} 189 \mathrm{r}^{\circ}\right)$; 1811 A Tour in Italy ; dernier cahier $\left(\mathrm{f}^{\circ} 190 \mathrm{r}^{\circ}\right) ; 1811$ Italie $\left(\mathrm{f}^{\circ} 190 \mathrm{v}^{\circ}\right)$; dernière partie $d u$ Journal ; second séjour à Milan ( $\left.\mathrm{f}^{\circ} 192 \mathrm{r}^{\circ}\right)$. Corrections et notes de régie de 1813 .

8. Voir Gérald Rannaud, «Le Journal de voyage, forme littéraire ou fait culturel ? », Le Journal de voyage et Stendhal, éd. Victor Del Litto et Emmanuel Kanceff, CERVI, Genève, Slatkine, 1986.

9. Nous reproduisons ces clichés avec l'aimable autorisation de la Bibliothèque municipale de Grenoble.

10. Almuth Grésillon, La Mise en auvre. Itinéraires génétiques, Paris, CNRS Éditions, 2008, p. 26-27.

11. Les numéros de registre renvoient désormais, sauf mention contraire, au catalogue des manuscrits de la bibliothèque municipale de Grenoble. 


\section{Les copies de 1813}

- R 5896 (2) Rés., (fos 162-175) : dictée de la partie manquante correspondant au voyage de Florence à Rome et au séjour à Rome ; chapitre « Musique à Naples »;

-1 re copie : cahier $\mathrm{n}^{\circ} 51$ (disparu) et cahier $\mathrm{n}^{\circ} 50$ (Manuscrit Arbelet : «Fin du Tour d'Italie ») ;

- transcription par A. Cordier du cahier $\mathrm{n}^{\circ} 50$ : copie Cordier (R 90729 (2), Rés.) ;

- 2e copie (mise au net) : copie Crozet-Royer «Tour d'Italie en 1811 par M. de Léry » (aujourd'hui introuvable).

\section{La campagne de relecture (1813)}

En janvier 1813, Stendhal rentre, épuisé, désespéré, malade, de la campagne de Russie. En mars, avec évidemment plus de distance personnelle et plus de conscience de sa vocation d'écrivain 12 , il reprend son manuscrit de 1811. Que fait-il alors ?

1. Il comble certaines lacunes de fragments perdus en cours de voyage, en dictant à son copiste quelques pages sur le trajet Florence-Rome et le séjour à Rome ainsi que la traduction de l'ouvrage de Luigi Galanti, Napoli $e$ il suo contorno (Naples et ses environs) qui formera le chapitre "Musique à Naples »; il relit cette copie et la corrige (R 5896, (2) Rés., fos $162 \mathrm{r}^{\circ}-175 \mathrm{v}^{\circ}$ ).

2. Il corrige son manuscrit de 1811 et le «prépare » pour la copie qu'en doit faire son copiste. On trouve donc des notes de régie destinées au copiste pour qu'il intercale des ajouts au bon endroit : « J'ai oublié l'autre registre dans la diligence. Placer ceci après la page $55 \mathrm{du}$ 1er cahier » (R 5896 (23) Rés., f ${ }^{\circ} 23$ r $^{\circ}$ ).

3. Il dicte à son copiste Fougeol cette version corrigée du manuscrit autographe en y intégrant la dictée de la copie des pages perdues. Il corrige cette copie (cahiers $\mathrm{n}^{\circ} 51:$ : commencement du voyage » et $50:$ : Fin du voyage $13 »)$ : cahiers $n^{\circ} 51$ (disparu) et cahier $n^{\circ} 50$ : copie Arbelet retrouvée en 2008.

4. Il dicte à nouveau à son copiste cette nouvelle mouture pour la mettre au net en y insérant les corrections et les ajouts transcrits sur la copie précédente, mais aussi d'autres modifications qu'il invente spontanément en dictant oralement (copie dite Crozet-Royer que Victor Del Litto dit avoir consultée chez Mme de Royer à Claix (Isère) ${ }^{14}$ ).

L'analyse des différentes strates du manuscrit nous renseigne de manière très précise sur le travail d'écriture stendhalien, déjà analysé ailleurs par Gérald Rannaud ${ }^{15}$ : à partir des corrections apportées sur le premier jet autographe, Stendhal invente oralement son texte par des dictées successives qu'il annote une fois qu'elles sont établies par son copiste et qu'il dicte à nouveau jusqu'à la dernière couche d'écriture. On voit ici que toute linéarité est exclue du processus d'écriture et que le « texte » se construit dans le mouvement de cette alternance rédaction-relecture, englobant successivement les annotations antérieures et les dépassant par l'improvisation orale (voir fig. 3).

\section{Vers une fictionnalisation du récit de soi ?}

Le travail de reprise du journal de 1811 doit nous conduire à en retrouver le sens.

Plusieurs niveaux de transformation sont à identifier : d'abord, les surcharges et ratures sur le manuscrit de 1811 vont, semble-t-il, dans le sens d'un effacement progressif des traces de subjectivité et de datation des entrées du journal, marques de l'écriture diariste : les noms propres sont remplacés par des initiales ou des pseudonymes ; les dates de rédaction du manuscrit autographe sont mises entre parenthèses pour que le copiste ne les reporte pas

12. En juillet 1812, il est parti pour la campagne de Russie avec les treize registres de l'Histoire de la peinture en Italie, son Journal de 1807 et Letellier, dans l'espoir de travailler.

13. Ces indications de la main de Romain Colomb, le cousin de Stendhal et son premier éditeur posthume, concernant la numérotation des cahiers de cette strate de copie de 1813, se trouvent sur la page de garde du volume R 5896 (23) Rés., f ${ }^{\circ} 1$.

14. Euvres intimes, t. I, op. cit., p. 1419.

15. Gérald Rannaud, «Éditer l'inachevé. Notes sur une édition du manuscrit de la Vie de Henry Brulard de Stendhal », Éditer des manuscrits. Archives, complétude, lisibilité, études réunies et présentées par Béatrice Didier et Jacques Neefs, Saint-Denis, Presses universitaires de Vincennes, coll. « Manuscrits Modernes », 1996, p. 133-158. 


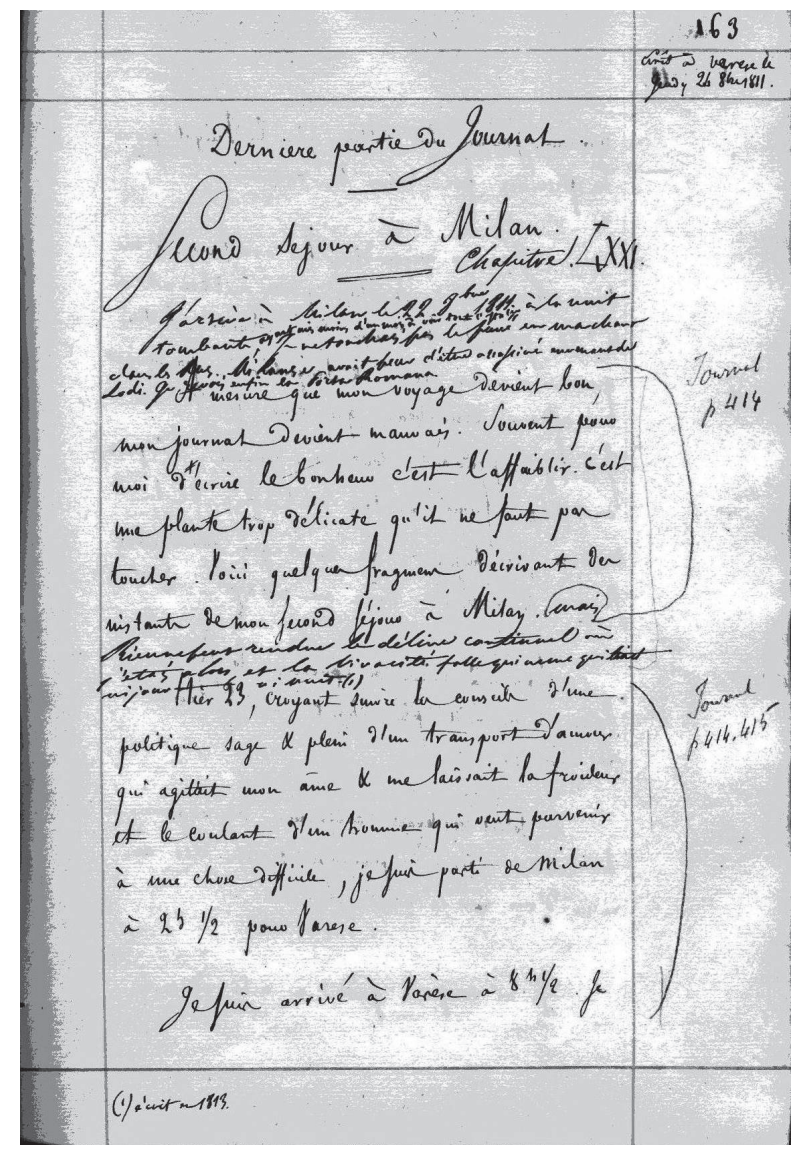

Fig. 3 : Manuscrit Arbelet, $\mathrm{f}^{\circ} 163 \mathrm{r}^{\circ}$ (coll. particulière)

sur la copie ; un découpage en chapitres est esquissé, en interligne, même si la numérotation de ces chapitres n'est pas systématique : parfois un blanc succède à la mention « chapitre », en vue d'un remaniement ultérieur (voir fig. 4).

La première copie de 1813 introduit du liant entre les chapitres (qui sont créés et numérotés de façon plus systématique que dans la version corrigée de 1811) afin d'effacer l'impression de discontinuité des entrées du journal. Par exemple, au folio $192 \mathrm{r}^{\circ}$ du volume XIV (R 5896 Rés.), on pouvait lire la remarque suivante :

À mesure que mon voyage devient bon mon journal devient mauvais. Souvent pour moi décrire le bonheur c'est l'affaiblir. C'est une plante trop délicate qu'il ne faut pas toucher. Voici quelques fragments décrivant des instants de mon second séjour à Milan.

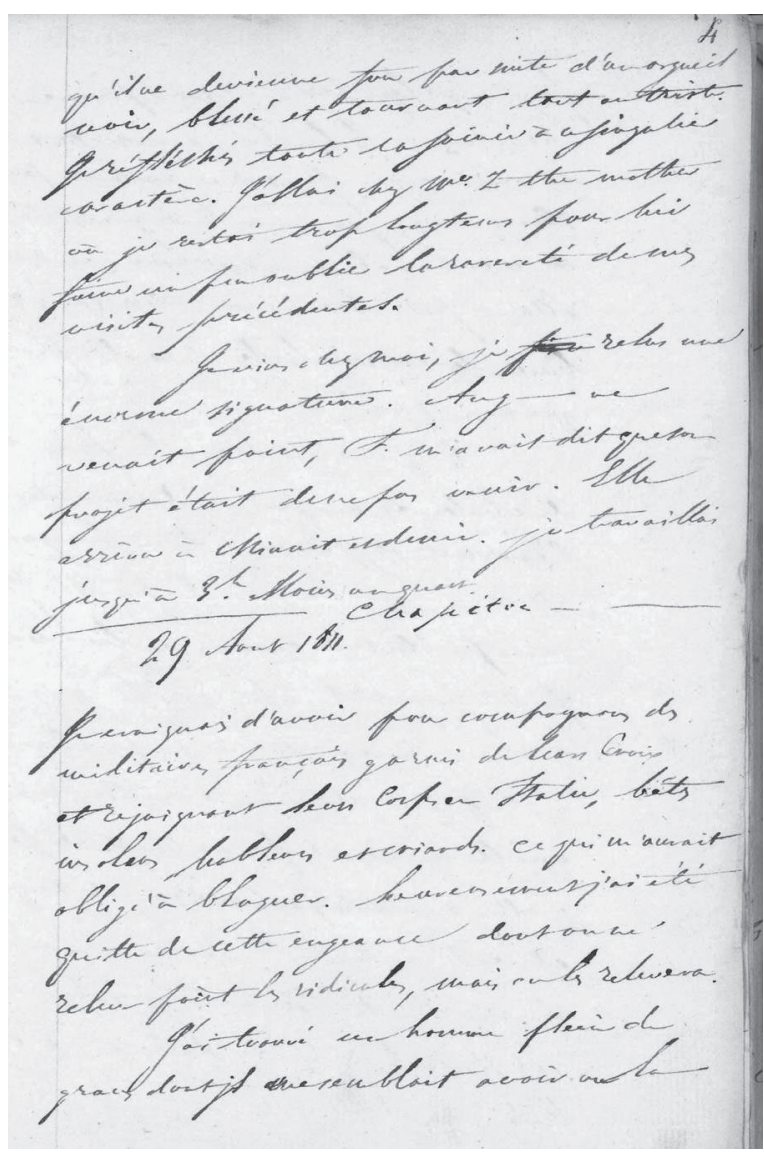

Fig. 4 : «A Tour Throught Italy. 1811 », $\mathrm{f}^{\circ} 4 \mathrm{r}^{\circ}$ (R 5896 (23) Rés., Bibliothèque municipale de Grenoble)

Sur le folio $163 \mathrm{r}^{\circ}$ du Manuscrit Arbelet (fig. 3), on peut lire l'ajout suivant, en interligne :

Chapitre LXII

J'arrivai à Milan le 22 8bre [22 octobre] 1811 à la nuit tombante ayant mis moins d'un mois à voir toute l'Italie. Je ne touchais pas le pavé en marchant dans les rues. Milanese avait peur d'être assassiné en venant de Lodi. Je revois enfin la Porta Romana.

À mesure que mon voyage devient bon $[\ldots]$.

Sont ajoutées les circonstances du retour à Milan et les impressions de bonheur qui s'y rattachent. Le récit devient plus factuel et un lien s'établit avec l'ensemble des pages écrites pour leur donner une cohérence globale plus visible : le journal se réécrit avec le savoir de ce qui s'est passé ultérieurement, ce qui est contraire à la logique 
propre du journal quotidien, inscient des événements du lendemain. Le récit au passé se substitue donc au discours du présent ; l'ajout est daté : « écrit en 1813 ».

Mais c'est sur la dernière copie du dossier manuscrit (copie Crozet-Royer) que les changements sont le plus caractéristiques : la première personne du singulier propre à l'écriture diariste cède la place à un narrateur, M. de Léry, qui aurait communiqué au narrateur premier son journal, selon la mise en scène éditoriale du manuscrit trouvé ou légué, typique des récits du XVIII siècle :

Tour d'Italie en 1811 par M. de Léry.

M. de Léry, capitaine aide de camp, m'a permis, avant de partir, de prendre ce que je voudrais dans ses manuscrits. J'ai fait copier un morceau sur le style et sa course en Italie. Il y a dans le récit de celle-ci plusieurs parties qui ne sont apparemment intelligibles que pour lui ${ }^{16}$.

Le récit est donc emboîté dans une fiction éditoriale, ce qui accroît encore la distance avec l'écriture au jour le jour du journal. La «Préface » de M. de Léry narrativise le processus d'écriture : elle explique comment le récit de 1811 sera gardé intact, dans la gangue du corps principal du manuscrit, mémoire des moments sacrés du bonheur d'avant 1813 ; les réflexions de 1813 seront, elles, détachées matériellement du texte, « en note », mais intégrées au discours préfaciel :

\section{Préface : Non sum qualis eram.}

Je suis malheureusement loin d'être l'homme de 1811. Je ne corrigerai donc rien à mes journaux de 1811. Ils perdraient en ressemblance à mes sensations ce qu'ils pourraient gagner en clarté et en agrément. Je suis dans le calme le plus parfait. À mon retour de Moscou, je n'ai plus retrouvé les passions qui animaient ma vie. [...] La froideur où je suis tombé actuellement ne serait pas désagréable, si j'avais le souvenir du bonheur que me donnaient les goûts qui occupaient ma vie avant mon voyage en Russie. [...]

Je viens de décacheter les cinq ou six enveloppes qui renfermaient le journal de 1811. Je vais le copier ou le faire copier exactement. Je ne me permettrai d'autre altération que de mettre en français les passages écrits alors en anglais pour la prudence. Tout ce que j'ajouterai sera en note et date de ce temps de froideur (1813) ${ }^{17}$.

Les notes ne sont donc plus des ajouts ou des corrections destinés à commenter le manuscrit de 1811, mais une autre voix dialoguant avec un autre temps de l'écriture et partie intégrante de cette nouvelle mouture qui englobe les deux strates de l'écriture. L'écriture agit donc par intégration de ce qui n'était qu'apostille et marginale ; elle se situe à l'intersection d'un temps mort dont il faut garder la mémoire que le narrateur a perdue et d'un temps présent, nostalgique de ces temps révolus.

Dans cette strate de 1813, la relation au lecteur est très perceptible alors que le diariste de 1811 hésitait à réserver l'écriture à son seul usage ou bien à la partager avec un éventuel lecteur, déclarant par exemple, à Milan, le 8 septembre 1811 : «Dirais-je ce qui m’a ému le plus en arrivant à Milan ? (on va bien voir que ceci n'est écrit que pour moi.) C'est une certaine odeur de fumier particulière à ces rues. Cela, plus que tout le reste, me prouvait apparemment que j'étais à Milan18. » Dans cet aveu, perçait déjà la présence d'un destinataire paradoxal, nié et sollicité à la fois : en effet, à qui s'adresse la question posée ? quel est ce « on » à qui elle est destinée ? Le «narrateur» de 1811 envisageait même parfois la présence effective d'un lecteur : «Je ne dis pas que ces jugements soient vrais pour vous qui me lisez, mais ils le sont pour moi, M.H.B., né en 1783 et ballotté par onze ans d'expérience. Voilà ce que je pense ${ }^{19}$. » Les copies de 1813 vont donc dans le sens d'une affirmation de plus en plus nette du caractère fictionnel du récit : le lecteur $\mathrm{y}$ est admis comme une instance évidente du processus énonciatif et le voyageur donne par avance à ses notes un destinataire, sans avoir besoin de le nommer précisément. Il lui offre, d'emblée, le mode d'emploi de son récit : «Je ne me permettrai d'autre altération que de mettre en français les passages écrits alors en anglais pour la prudence. Tout ce que j'ajouterai sera en note et date de ce temps de froideur (1813). »

Les différentes strates du dossier manuscrit nous montrent également les mutations génériques de cette écriture en devenir. En 1811, malgré elle, à la faveur

16. Euvres intimes, t. I, op. cit., p. 1422.

17. Ibid.

18. Ibid., p. 736. Voir aussi ibid., p. $805:$ « Ce journal est fait pour Henri, s'il vit encore en 1821. Je n'ai pas envie de lui donner occasion de rire aux dépens de celui qui vit aujourd'hui. Celui de 1821 sera devenu froid et plus haïssant. »

19. Ibid., p. 787. 
d'un événement imprévu, l'écriture du journal est déviée de ses fonctions initiales (journal de voyage) et dérive vers l'« intime »: Stendhal tombe amoureux d'Angela Pietragrua. Ainsi, entre le moment qui précède le premier séjour à Milan et celui qui lui succède, la tonalité change du tout au tout : le Tour d'Italie n'est plus vraiment le but du voyage ; depuis qu'il a quitté Milan le 21 septembre, le narrateur n'a plus qu'une obsession, retrouver Angela. Le 9 octobre, à Naples, il écrit son impatience :

Je crois que je suis amoureux de [Mme Pietragrua]. Du moins, depuis Bologne, $\mathrm{j}$ 'aurais toujours mieux aimé être avec elle qu'au lieu où je me trouvais. Je me surprends sept à huit fois par jour à penser à elle avec tendresse, avec rêverie ; ma respiration est accélérée et je quitte avec peine ce doux penser. [...] Il me semble que ce que j'éprouve est de l'amour dans toute l'étendue que ce mot a dans mon esprit. Je grille de retourner à Milan. Rien ne m'émeut. Je serais plus sensible à ce que je vois si j'eusse sauté Milan. Peut-être n'irai-je pas à Ancône20.

Mais, de manière concurrente, au cours du voyage, une autre vocation se dessine et l'artiste se révèle sous l'amoureux :

J'ai cru longtemps être né insensible à la sculpture et même à la peinture.

Mais enfin près d'Iselle, en sentant le genre de cascade qu'il conviendrait de mettre autour du palais de La Pauvreté, j'ai compris que je sentais aussi le langage des choses muettes ${ }^{21}$.

À la toute fin du journal de 1811, au moment même où l'écriture s'épuise, c'est même la seule orientation qui persiste, sous forme de bribes discontinues, de notations elliptiques. Et le « Tour d'Italie » s'achève ainsi :

7 novembre 1811

Vu ce matin la galerie de l'Archevêché. Belle figure de Procaccini.

Copie de la Madeleine du Corrège qui me semble jolie.

Beau portrait de pape, en petit de Titien, dit-on.

Relief d'un profil du Titien²2.

Le «Voyage en Italie » est donc non seulement un brouillon jamais publié mais un inachevé, un récit ouvert, tendu vers autre chose que l'écriture du journal. À un moment donné, Stendhal a sans doute conscience que son Journal de voyage est devenu une esquisse différente de ce qu'il avait imaginé qu'il pouvait être ; ou bien peut-être le voyage - et son écriture quasi quotidienne - donne-til un autre élan à son désir d'écriture. L'épuisement que nous constatons à la fin de ce cahier est paradoxalement un processus dynamique : Stendhal abandonne certes son manuscrit, pratique fréquente chez lui, mais ni définitivement, ni absolument, comme on va le voir.

La copie Arbelet répare en effet cette fin trop abrupte en lui adjoignant un nouveau chapitre (LXXX) qui ferme de manière moins soudaine le processus d'écriture et qui, par l'ajout du mot « Fin », ôte le caractère nécessairement inachevé du journal non publié :

\section{[Chapitre LXXX}

Après cela je fus trop heureux et trop occupé par la jalousie de ces MM. pour avoir le temps d'écrire. Je partis de Milan le 13 novembre, arrivai à Paris le 27 novembre à 5 h 1/2. Great. Le lendemain bataille perdue.

Fin.]

La boucle du voyage est donc bouclée : la mention du retour à Paris en signe l'achèvement par une clôture artificielle (puisque ajoutée après-coup et non en temps et lieu du voyage, voir fig. 5).

Sur la copie Crozet-Royer, la mise en récit est accentuée. La mention «Chapitre I » remplace la date liminaire, intégrée désormais au flux de la narration : «Hier, 25 août 1811, j'ai arrêté ma place dans la diligence de Milan. » L'effet de localisation et de datation propre au « Journal » est donc effacé au profit d'un lissage des aspérités produites par la narration chaotique et incertaine de l'écriture journalière. L'alternance imparfait/passé simple renforce l'homogénéité du récit rétrospectif. D'autre part, le départ, loin d'être marqué par l'émotion des adieux, comme en 1811, est motivé par des raisons psychologiques et esthétiques (déjà s'amorce ici la ligne de partage entre l'Italie, contrée de toutes les délices, et la France, terre d'ennui, qu'on retrouvera comme une constante dans les écrits postérieurs de Stendhal).

Je serai à Milan dans dix jours. Je suis allé à Versailles en une heure un quart dans mon cabriolet. J'y vis la pièce du Dragon.

20. Ibid., p. 794-795.

21. Ibid., p. 736.

22. Ibid., p. 815. 


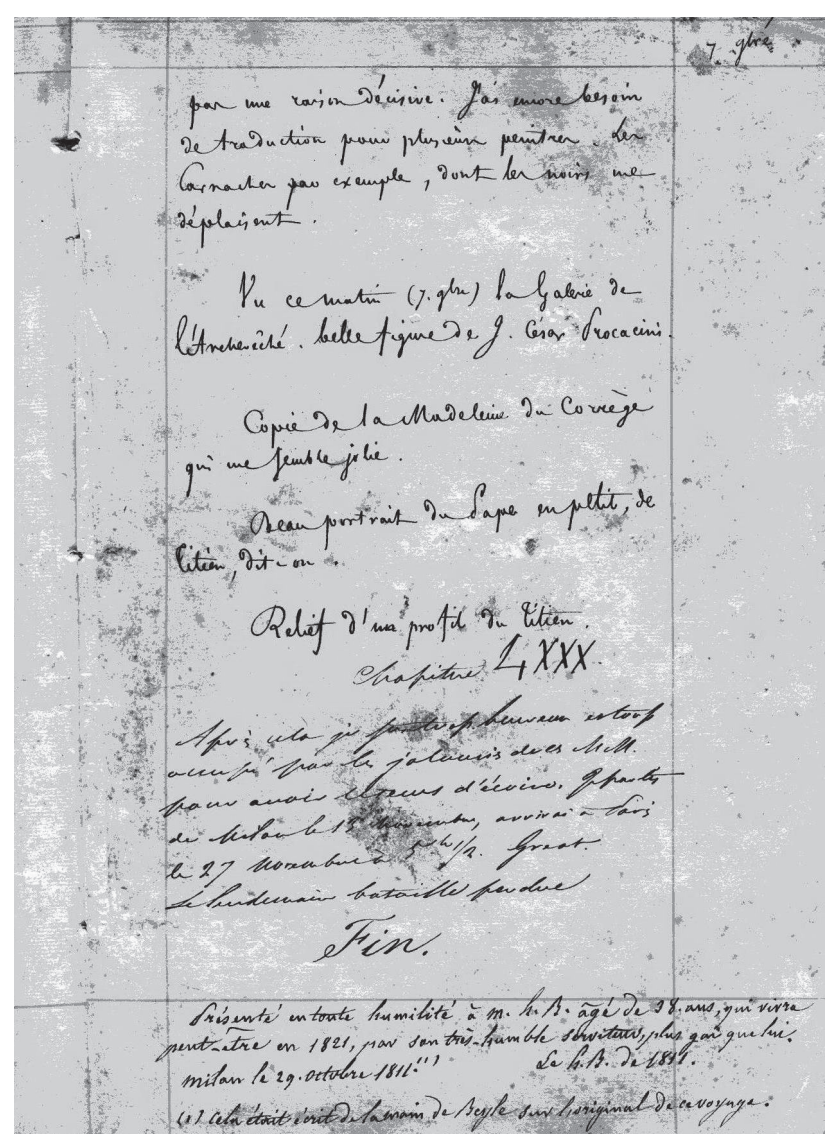

Fig. 5 : Manuscrit Arbelet, $\mathrm{f}^{\circ} 195 \mathrm{r}^{\circ}$ (coll. particulière)

Tout cela m'a paru plat, petit et ennuyeux. Parfaitement heureux à Paris, j'en suis dégoûté à fond ${ }^{23}$.

On constate une nette orientation du dossier manuscrit vers un stade de condensation à la fin du processus de rédaction. Les copies de 1813 tendent en effet à une littérarisation progressive par rapport au support premier du journal. Il existe, certes, une intention littéraire à la source du projet (nous l'avons vu dans la manière d'isoler d'emblée le journal de voyage en unité indépendante pourvue d'un titre) mais qu'elle soit dès le départ tournée vers un projet de publication, comme le prétend Victor Del Litto ${ }^{24}$, me semble fausser la temporalité particulière de l'entreprise et renoncer à «écouter » ce que nous disent les vagues successives du manuscrit. Qu'un
« journal »soit, deux ans après son écriture spontanée et discontinue, retravaillé, retouché, comme un brouillon ordinaire montre, je crois, que quelque chose a changé, qu'une autre visée s'est superposée à la première, qu'une réorientation s'est imposée en cours d'élaboration. À partir du moment où l'écrivain intervient sur l'écriture première, où il paye des copistes pour effectuer un véritable travail de ressaisie et de transformation du premier jet manuscrit, on n'est plus dans le « journal intime » écrit en voyage. Le dossier génétique du Tour through Italy nous montre, par son inflexion vers une narration de moins en moins improvisée, que la réutilisation des éléments du journal s'engage dans une voie nouvelle qui rompt avec ce qui précède et s'éloigne de la perspective diariste. La visée téléologique qui consiste à assigner un rôle prédéterminé à tout écrit est défaite par l'étude transversale d'un processus d'écriture : le Henri Beyle de 1813, qui n'est pas encore Stendhal, rappelons cette évidence, cherche à écrire, mais il ne sait pas encore quels seront le sens et le genre de cette écriture : le théâtre ? l'essai philosophique ou esthétique ? pourquoi pas l'écriture de voyage, alors «dans le vent »? Il sent qu'il y a, dans ce journal de 1811 une matière prometteuse dont il pourrait tirer quelque chose mais qui n'est encore, en 1813, qu'une interrogation, une substance informe qui cherche à prendre consistance, tournée vers l'avenir d'une écriture possible. L'étude minutieuse du manuscrit nous contraint à ce travail d'observation dans l'ordre de la chronologie : le journal de 1811 est devenu un manuscrit de travail, c'est-à-dire un brouillon ouvert à des transformations à venir. De fait, on va le voir, Stendhal, dans ses premiers pas d'écrivain, et même beaucoup plus tard, pioche dans cette réserve d'écriture.

En effet, on s'aperçoit que le retour d'Italie en novembre 1811 coïncide avec le début de la rédaction de l'Histoire de la peinture en Italie qui sera mis en vente en 1817. Le diariste avait indiqué à la fin du Tour qu'il

\section{Ibid., p. 1423}

24. Victor Del Litto soutient qu'en 1813 « le projet a germé dans [1']esprit [de Stendhal] de publier le Tour through Italy » et commente ainsi l'abandon du manuscrit : «C'est pourquoi Stendhal, toute réflexion faite, a eu raison de renoncer à son projet de publication », « Journal de voyage et journal intime chez Stendhal », Le Journal de voyage et Stendhal, op. cit., p. 10-11. 
commençait à lire Lanzi, critique d'art italien qu'il traduit et dont il copie de larges extraits dans l'Histoire de la peinture en Italie. La première impulsion de ce projet abouti se situait à la fin du manuscrit de 1811. En 1814, Stendhal rédige les Vies de Haydn, Mozart et Métastase qu'il met en vente en 1815 (c'est son premier livre publié). Or les premières pierres de cet ouvrage sont jetées dans le chapitre « Musique à Naples » que Stendhal réintroduira quasiment sans retouches dans le texte de 181525. En septembre 1817, Rome, Naples et Florence en 1817 est mis en vente : en mars de la même année, Stendhal note sur le manuscrit de 1811 : «Bon livre à traduire en français en 2 volumes » : il recycle donc certaines pages du Tour through Italy en 1811 pour Rome, Naples et Florence en 1817 et ce livre gardera l'ossature du carnet de route de 1811, avec des modifications dues à la situation personnelle de l'auteur et à la position de l'Italie après la chute de l'Empire. Le narrateur est devenu «M. de Stendhal, officier de cavalerie 26 » - une réminiscence de $\mathrm{M}$. de Léry « capitaine aide de camp »-, le caractère intime du journal de 1811 est très atténué mais l'effet-journal demeure (datation, localisation quotidiennes).

Entre 1815 et 1817 , commence donc à se constituer un corpus italien correspondant aux premières publications de Stendhal dont la matrice est sans doute le Tour through Italy. Plus tard, dans L'Italie en 1818 , dans Rome, Naples et Florence (1826) et même dans les Promenades dans Rome (1829), on retrouve des échos de ce premier noyau.

\section{Conclusion et suggestions}

Les « genèses » du Tour through Italy ne conduisent donc directement à aucune « œuvre ». Elles sont des pistes, explorées dès 1813 par Stendhal à partir de la matière informe du journal de 1811 et reprises à plusieurs occasions de rédaction sur le sujet italien : le journal lègue alors son allure improvisée aux récits de voyage ultérieurs et oriente leur contenu. L'étude $\mathrm{du}$ dossier manuscrit nous permet ainsi de percevoir la linéarité brisée d'une écriture qui ne sait où elle va mais qui a compris que l'obstination à transformer le premier jet pouvait conduire à une constellation de textes. C'est ce mouvement imprévisible que nous avons voulu restituer et qui éclaire aussi de manière intéressante le processus de création de Stendhal : que ce soit, plus tard à la fin des années 1830 , pour la Vie de Henry Brulard ou pour les Mémoires sur Napoléon27, différentes étapes des brouillons sont gardées en réserve en vue d'une écriture plus élaborée, sans qu'un projet précis leur soit nécessairement assigné au départ. Cette écriture-là est donc générative : elle s'enroule sur ellemême jusqu'à se figer dans une version satisfaisante (ou pas, la plupart des écrits de Stendhal restant à l'état de brouillon avancé, même s'ils furent publiés de manière posthume comme des œuvres achevées). Le dossier qui nous intéresse ici est donc un travail préparatoire, un matériau disponible ouvert sur d'autres territoires.

Il est évident que la meilleure manière de rendre lisible les différentes strates du dossier manuscrit que je viens de présenter est la publication en ligne. Un tel type d'édition permet de rendre concomitants les divers états du brouillon sans « écraser » aucune de ses formes existantes : c'est ce qui est prévu, selon le protocole établi par l'équipe «Manuscrits de Stendhal » de l'université de Grenoble, grâce au logiciel Morphon sur lequel nous travaillons et qui reproduit les manuscrits selon une transcription quasi diplomatique 28 . Pour cette transcription, les différentes versions du manuscrit s'afficheront, à la demande de l'utilisateur, en vis-à-vis de l'image correspondante en fac-similé, sans aucune hiérarchie préétablie.

Nous espérons ainsi représenter l'inachèvement dynamique d'un brouillon resté sous tension.

25. Lettre XIV sur Haydn, Vies de Haydn, Mozart et Métastase, recueilli dans L'Âme et la musique, éd. Suzel Esquier, Paris, Stock, 1999, p. 93-94. 26. Stendhal, Voyages en Italie, éd. Victor Del Litto, Paris, Gallimard, coll. «Bibliothèque de la Pléiade », 1973, p. 1.

27. Voir mon introduction à Napoléon de Stendhal, Paris, Stock, 1998.

28. Pour de plus amples informations, on peut consulter le site : <www. manuscrits-de-stendhal.org $>$. Cette édition électronique en ligne, en collaboration avec la bibliothèque municipale de Grenoble, sera doublée d'une édition papier dont les deux premiers volumes paraîtront aux ELLUG (Grenoble) en 2011, sous le titre Journaux et papiers de Stendhal, éd. Cécile Meynard, Hélène de Jacquelot et Marie-Rose Corredor ; les deux volumes suivants, incluant A Tour through Italy, verront le jour en 2012, chez le même éditeur, éd. Catherine Mariette, Cécile Meynard, Hélène Spengler et Elaine Williamson. 
Catherine Mariette-Clot est maître de conférences en littérature française à l'université de Grenoble, membre de l'équipe E.A. 3748. Une grande partie de sa recherche est consacrée à l'œuvre de Stendhal dont elle a édité la Vie de Napoléon et les Mémoires sur Napoléon (Napoléon de Stendhal, Paris, Stock, 1998). Elle se consacre actuellement, en collaboration avec l'équipe «Manuscrits de Stendhal » et la bibliothèque municipale de Grenoble, à l'édition électronique et papier (ELLUG, 2012) des Journaux et papiers de Stendhal.

Catherine Mariette-Clot, cath.marietteclot@free.fr

Résumés

Genèses du Tour through Italy de Stendhal (1811-1813)

La découverte au printemps 2008 d'une des copies de 1813 du Tour through Italy (1811), corrigée de la main de Stendhal, est à l'origine d'une volonté de reconstituer les différentes strates d'un dossier génétique presque complet ; deux ensembles s'en dégagent : un autographe de 1811, simple journal de voyage, censé consigner des événements advenus au jour le jour ; trois séries de copies, retravaillées en 1813, et dont la destination est sans doute de constituer un réservoir d'écriture pour des publications à venir. Dans Rome, Naples et Florence en 1817, L'Italie en 1818, Rome, Naples et Florence (1826), Promenades dans Rome (1829), on retrouve en effet des « extraits » de cette matière première de 1811-1813. Cet exemple nous permet d'avancer une hypothèse sur la méthode de composition stendhalienne : le «Journal » n'est pas seulement une écriture pour soi mais une sorte d'hypotexte toujours susceptible d'être modifié.

The discovery in 2008 of an 1813 copy of the Tour through Italy (1811) with handwritten corrections by Stendhal himself led to the project to piece together the various layers of a practically complete genetic dossier. Two sets emerge: an 1811 autograph manuscript, a mere travel diary, for jotting down daily events; three series of copies, reworked in 1813, which were probably meant to constitute a provision of texts for future publications. Indeed Rome, Naples et Florence en 1817, L'Italie en 1818, Rome, Naples et Florence (1826), Promenades dans Rome (1829), contain extracts from this 1811-1813 raw material. This example enables us to form a hypothesis on Stendhal's composition method: the "Journal" is not only a writing for himself but also a type of hypotext always modifiable.

Eine der aus dem Jahre 1813 stammenden Fassungen der Tour through Italy (1811) - von der Hand Stendhals korrigiert und im Frühjahr 2008 entdeckt - löste den Wunsch nach einer Rekonstitution der verschiedenen Schichten eines fast vollständigen textgenetischen Dossiers aus; es besteht aus zwei Einheiten: eine Handschrift von 1811, ein einfaches Reisetagebuch, das der Aufzeichnung tagtäglich sich ereignender Vorkommnisse gewidmet war; drei Fassungen von Abschriften, die im Jahre 1813 überarbeitet wurden und deren Bestimmung wahrscheinlich darin lag, als Schreibreservoir für zukünftige Publikationen zu dienen. Tatsächlich findet man in Rome, Naples et Florence en 1817, L'Italie en 1818, Rome, Naples et Florence (1926), Promenades dans Rome (1829) Auszüge aus diesem Rohmaterial von 1811-1813. Dieses Beispiel erlaubt uns, eine Hypothese über Stendhals Kompositionsmethode aufzustellen: Das „Tagebuch“ ist nicht nur dem Schreiben „für sich“ vorbehalten, sondern stellt eine Art Hypotext dar, der jederzeit modifiziert werden kann.
E1 descubrimiento, en la primavera de 2008, de una de las copias de 1813 de Tour through Italy (1811), con correcciones manuscritas de Stendhal, motivó el proyecto de reconstituir los diferentes estratos de un dossier genético casi completo; dos conjuntos se distinguen: un documento autógrafo de 1811, simple diario de viaje, destinado a registrar los sucesos ocurridos día a día, y tres series de copias, reelaboradas en 1813, y cuyo objetivo es sin duda constituir una reserva de escritura para publicaciones futuras. En Roma, Nápoles y Florencia en 1817, Italia en 1818, Roma, Nápoles y Florencia (1826), Paseos en Roma (1829), aparecen, en efecto, "fragmentos" de esa materia prima de 1811-1813. Este ejemplo nos permite formular una hipótesis acerca del método de composición stendhaliano: el "Diario" no constituye solamente una escritura para sí mismo sino una suerte de hipotexto susceptible de ser modificado en cualquier momento.

A descoberta na primavera de 2008 de uma das cópias de 1813 da Tour through Italy (1811), corrigida pela mão de Stendhal, está na origem do desejo de reconstituir os diferentes estratos de um dossier genético quase completo. Dois conjuntos se evidenciam: um autógrafo de 1811, simples diário de viagem, destinado a consignar acontecimentos ocorridos no dia a dia; três séries de cópias, retrabalhadas em 1813, e destinadas sem dúvida a constituir um reservatório de escrita para publicações futuras. Em Rome, Naples et Florence en 1817, L'Italie en 1818, Rome, Naples et Florence (1826), Promenades dans Rome (1829), encontram-se com efeito "extractos" dessa matéria prima de 1811-1813. Exemplo que nos permite avançar uma hipótese sobre o método de composição stendhaliana: o "Diário" não é apenas uma escrita para si mas uma espécie de hipotexto sempre susceptível de ser alterado.

La scoperta, nella primavera 2008, di una delle copie del 1813 del Tour through Italy (1811), corretta per mano di Stendhal, è all'origine di una volontà di ricostituire i differenti strati di un fascicolo genetico pressoché completo. Ne scaturiscono due insieme: un autografo del 1811, semplice diario di viaggio, nel quale sono consegnati gli avvenimenti giorno per giorno; tre serie di copie, rielaborate nel 1813 , e destinate a costituire una riserva di scrittura per pubblicazioni successive. In Rome, Naples et Florence en 1817, L'Italie en 1818, Rome, Naples et Florence (1826), Promenades dans Rome (1829), si ritrovano infatti alcuni "estratti" di questa prima elaborazione del 18111813. Questo esempio ci permette di proporre un'ipotesi sul metodo di composizione di Stendhal: il "Diario" non è solamente scrittura per il suo autore ma una sorta di ipotesto sempre modificabile. 